\title{
COHESIVE AXONAL TRANSPORT OF THE SLOW COMPONENT b COMPLEX OF POLYPEPTIDES ${ }^{1}$
}

\author{
JUDY A. GARNER ${ }^{2}$ AND RAYMOND J. LASEK \\ Department of Anatomy and Neurobiology Center, Case Western Reserve University School of Medicine, \\ Cleveland, Ohio 44106
}

Received March 18, 1982; Revised June 7, 1982; Accepted June 10, 1982

\begin{abstract}
The axonal transport of the diverse group of polypeptides characteristic of slow component $b$ (SCb), or Group IV, may be accomplished either by a bulk flow mechanism acting on each of the individual polypeptides or by their movement through the axoplasm as part of a physical complex or structural assembly. In order to determine if the $\mathrm{SCb}$ polypeptides travel as a cohesive unit (which would be consistent with the latter alternative above), we examined in detail the quantitative distribution of the individual $\mathrm{SCb}$ polypeptides in the guinea pig optic system at three different times after pulse radiolabeling the neuron cell bodies. Eighteen $\mathrm{SCb}$ polypeptides were selected as appropriate for analysis. Individual optic systems at the three select times after labeling were harvested, cut into small segments, and subjected to SDS-PAGE. Individual radioactive polypeptide bands were identified and located by fluorography, excised, and then quantified by scintillation counting.

The results indicate that the $\mathrm{SCb}$ polypeptides travel cohesively: they all have nearly superimposable distribution profiles at the advancing "front" of the $\mathrm{SCb}$ wave of radioactivity. However, the distribution profiles are not identical: there is considerable variation among the polypeptides in the portion of the distribution profiles trailing behind the advancing peak and in the breadth of the peak's crest. This variation is consistent with the hypothesis that the individual polypeptides have differing affinities for an $\mathrm{SCb}$ group, or structure, as it moves down the axon.
\end{abstract}

The majority of material transported anterogradely in axons is transported within three distinct axonal transport components. These three components of axonal transport were characterized initially by the rate at which they traversed the axons (as in Grafstein and Forman, 1980); however, recently, the idea has emerged that they also may be structurally and functionally distinct entities (Droz et al., 1975; Lorenz and Willard, 1978; Lasek, 1980; Tytell et al., 1981). The fast component of axonal transport (moving at 250 to $400 \mathrm{~mm} /$ day) and the slower component (SCa, moving at 0.2 to $1 \mathrm{~mm} /$ day) have both been relatively well characterized by both rate determinations and macromolecular constituents (Ochs, 1972;

\footnotetext{
'We gratefully acknowledge the excellent technical assistance of Shirley Ricketts and Diane Filsinger, the assistance of Michael Lark with the computations, and the secretarial skills of Mrs. Ann Martin during the completion of this work. This study was supported by the National Institutes of Health Predoctoral Fellowship HD-07104 to J. A. G. and National Institutes of Health Grants NS 14900-02 and NS 13658-03 to R. J. L. This work has been presented in preliminary form (Garner and Lasek, 1978).

${ }^{2}$ Present address: Department of Chemistry, Brain Research Group, Indiana University, Bloomington, IN 47405.
}

Hoffman and Lasek, 1975; Willard et al., 1974; Willard and Hulebak, 1977; Tytell et al., 1981). The fast component contains primarily glycoproteins (Forman et al., 1972), membranes and membrane vesicles (Droz et al., 1975; Tsukita and Ishikawa, 1980), or the materials related to transmitter release (Schwartz et al., 1976; Brimijoin, 1975). SCa, on the other hand, is composed primarily of cytoskeletal proteins, including tubulin and the neurofilament-associated proteins (Hoffman and Lasek, 1975; Lasek and Hoffman, 1975). Its structural counterpart has been suggested to be the axonal cytoskeleton (Lasek, 1980).

The third major axonal transport component (at 2 to $4 \mathrm{~mm} /$ day) is the component designated $\mathrm{SCb}$ (Black and Lasek, 1979, 1980; Brady and Lasek, 1981; Garner and Lasek, 1981) or the Group IV polypeptides (Willard et al., 1974). SCb is extremely complex in constitution, the identity of its constituents ranging from microfilamentassociated proteins, such as actin (Black and Lasek, 1979; Willard et al., 1979), the myosin-like M-2 protein (Willard, 1977), and clathrin (Garner and Lasek, 1981), to many of the metabolic enzymes, such as creatine phosphokinase, nerve-specific enolase, and calmodulin (Brady and Lasek, 1981; Brady et al., 1981). 
This last observation is particularly interesting in that the metabolic enzymes traditionally have been viewed as "soluble proteins." The question that naturally arises is how the axonal transport of these soluble proteins, in the company of known structural proteins, is actually accomplished. There are two alternative modes of axonal transport that have been suggested. First, the soluble proteins could move independently, and their transport would thus be a result of "bulk axoplasmic flow" (Weiss and Hiscoe, 1948; Gross and Beidler, 1975; Cancalon and Beidler, 1975). The alternative view would be that the soluble and structural proteins do not move independently, but rather as part of one or more structural assemblies (Brady and Lasek, 1981; Brady et al., 1981; Lasek, 1980; Garner and Lasek, 1981; Tytell et al., 1981).

'These two perspectives would lead to predictions concerning the detailed behavior of the individual $\mathrm{SCb}$ proteins during their axonal transport. If the $\mathrm{SCb}$ proteins move totally independently, as a result of bulk flow, the kinetics of transport of individual proteins would only be constrained by their individual affinities for, or charge interactions with, more rapidly or more slowly moving materials (i.e., membranes or microtubules). Therefore, one would expect to see a physical separation of the different $\mathbf{S C b}$ polypeptides over distance, with the rate of movement of each polypeptide being a complex function of $(a)$ its response to "bulk flow" and $(b)$ its personal interactions with other constituents in the axoplasm. In other words, it would be very unlikely for many of the SCb polypeptides to have identical distribution kinetics with time. If, however, the "soluble" and structural $\mathrm{SCb}$ proteins are transported as part of one or more complexes or assemblies, one would expect to see extreme similarity among the distribution profiles, particularly in the region of the advancing "front."

In the present work, we have tested these two alternatives by analyzing quantitatively the distribution of individual radiolabeled $\mathrm{SCb}$ polypeptides in guinea pig retinal ganglion cell axons at different times during their axonal transport.

\section{Materials and Methods}

Radiolabeling polypeptides within the axons. The visual systems of adult, male Hartley strain (500 to 700 gm) guinea pigs were used for all experiments. The technique for injection of amino acids into the posterior chamber of the eye and thus radiolabeling axonally transported retinal ganglion cell proteins was that used by Garner and Lasek (1981). In all experiments, $10 \mu \mathrm{l}$ of a concentrated (45 to $48 \mu \mathrm{Ci} / \mu \mathrm{l}$ ) one-to-one mixture of tritiated amino acids $\left(4,5-\left[{ }^{3} \mathrm{H}\right](N) 1\right.$-lysine, 40 to $80 \mathrm{Ci} /$ mmol, New England Nuclear; and 2,3-[ $\left.{ }^{3} \mathrm{H}\right](N)$ 1-proline, 20 to $30 \mathrm{Ci} / \mathrm{mmol}$, New England Nuclear) was injected into either one or both eyes of each animal. Animals were anesthetized with ether at appropriate times after injection and sacrificed by decapitation, and visual systems were harvested by dissection.

SDS-polyacrylamide gel electrophoresis. Samples to be electrophoresed were solubilized in BUST ( $1 \%$ SDS, $5 \% \beta$-mercaptoethanol, $1 \mathrm{~mm}$ phenylmethylsulfonyl fluoride, $10 \mathrm{~mm}$ 'Tris, in $8 \mathrm{M}$ urea, at $\mathrm{pH} 6.8$ ) at $98^{\circ} \mathrm{C}$ for 1 to $2 \mathrm{~min}$. Sample electrophoresis was performed in slab gradient (4 to $17.5 \%$ ) polyacrylamide gels in the buffer system described by Laemmli (1970). Gels were stained with $0.1 \%$ Coomassie brilliant blue $\mathrm{R}$, destained, and fluorographed according to the method of Bonner and Laskey (1974) and Laskey and Mills (1975). Two-dimensional gel electrophoresis was performed as described by O'Farrell (1975).

SCb polypeptides. The SCb polypeptides selected for examination were those initially described by Black and Lasek (1980), as well as several less radioactive bands consistently present in the SCb polypeptide profile in the 4 to $17.5 \%$ polyacrylamide gel system. The molecular weights of the $\mathrm{SCb}$ polypeptides were assigned by their electrophoretic mobility relative to the following known molecular weight standards: vitellogenin $(240,000)$, thyroglobulin $(225,000,215,000)$, phosphorylase A $(94,000)$, bovine serum albumin $(68,000)$, and ovalbumin $(43,000)$. The relative amount of radioactivity in the $\mathrm{SCb}$ polypeptide bands was determined at 6 days after intraocular injection. Identical amounts of sample were electrophoresed in adjacent gel wells. The lanes were cut from the gels: one lane was fluorographed, and an identical lane was frozen and cut into $1-\mathrm{mm}$ segments with a Mickle gel slicer (Brinkman Instruments). The slices were dissolved in $0.5 \mathrm{ml}$ of $30 \% \mathrm{H}_{2} \mathrm{O}_{2}$ at $60^{\circ} \mathrm{C}$ overnight. Five $\mathrm{ml}$ of New England Nuclear Formula 963 aqueous scintillation cocktail were added to each slice before counting on a scintillation counter. Counting efficiency (30 to 31\%) was determined by internal standardization.

Analysis of the anterograde movement of the individual SCb polypeptides. In order to analyze the anterograde movement of the $\mathrm{SCb}$ polypeptides, animals were sacrificed at 4, 6, and 9 days after injection of labeled protein precursors into one or both of their eyes, and the distribution of radiolabeled polypeptides in sequential 1$\mathrm{mm}$ segments of optic nerve, chiasm, and tract was examined. Fluorographs were used as a guide to locate the individual SCb polypeptides in each gel profile. The appropriate band region in the gel was excised carefully and incubated in $0.5 \mathrm{ml}$ of $30 \% \mathrm{H}_{2} \mathrm{O}_{2}$ at $60^{\circ} \mathrm{C}$ overnight to dissolve the gel. New England Nuclear Formula 963 (5 ml) scintillation cocktail was added to each sample and the sample was counted. Counting efficiency ranged from 25 to $30 \%$; counts were corrected for quenching and converted to disintegrations per minute.

Since the amount of radioactivity incorporated into the retinal ganglion cells in each eye varies considerably, the data for "total $\mathrm{SCb}$ radioactivity distribution" and the radioactivity distribution for the individual polypeptides were standardized. First, the total $\mathrm{SCb}$ radioactivity was obtained by summing the radioactivity in all of the segments of the visual system from a particular preparation. The fraction of radioactivity contained in each segment was converted to a percentage of the total sum. The mean percentage of radioactivity in a particular segment was obtained by averaging with the radioactivity in the corresponding segments from other experimental preparations (based on their precise distance from the eye), and the standard error was computed. The distribution of the radioactivity for the individual polypeptides was obtained similarly. The total radioactivity in an individual polypeptide band throughout the visual system was determined by summing the amount of radioactivity in the band in each segment. The amount of 
radioactivity present in that band in each segment was then expressed as a percentage of the total. Again, the percentages for corresponding segments from all experimental animals were averaged and the standard error was computed. (The number of nerves and tracts analyzed was 8 at 4 days, 10 at 6 days, and 5 at 9 days.) The chiasm region was treated differently, as each segment of chiasm received radioactive proteins from both eyes. Therefore, the proportion of radioactivity in the chiasm segment was corrected by calculating the proportion of total radioactivity in one nerve and tract relative to the total radioactivity in the opposite nerve and tract.

Centrifugation and liquid chromatography of the $S C b$ polypeptides. Information about the properties of the $\mathrm{SCb}$ polypeptides under nondenaturant conditions was obtained by centrifugation and liquid chromatography on Sephadex G-200. Six days after intraocular injection, optic nerves and chiasma (containing only radioactive $\mathrm{SCb}$ polypeptides) were harvested. Tissue was combined with $1.0 \mathrm{ml}$ of buffer $\left(150 \mathrm{~mm} \mathrm{KCl}, 1.5 \mathrm{~mm} \mathrm{MgCl}_{2}, 0.5\right.$ mM EGTA, $10 \mathrm{~mm}$ imidazole, $1 \mathrm{~mm}$ phenylmethylsulfonyl fluoride, $40 \mu \mathrm{M} \mathrm{CaCl}_{2}, \mathrm{pH} 6.9$ ) at $4^{\circ} \mathrm{C}$ and homogenized with a glass/Teflon homogenizer. Samples were centrifuged at $114,000 \times \mathrm{g}$ for $60 \mathrm{~min}$ in a Beckman SW 65 rotor in a Beckman L2-65B ultracentrifuge. The pellet and the floating lipid layer were counted and electrophoresed on gels. A sample volume of $400 \mu \mathrm{l}\left(1\right.$ to $1.5 \times 10^{6}$ cpm, $800 \mu$ g protein) was chromatographed on a Sephadex G-200 column $(40 \mathrm{~cm} \times 1 \mathrm{~cm})$ with a flow rate of 6.5 $\mathrm{ml} / \mathrm{hr}$. One-milliliter fractions were collected and $20-\mu \mathrm{l}$ aliquots were counted. Recovery of radioactivity from the column was always $98 \%$ or better. Fractions had "cold" brain supernatant added as carrier (20 to $50 \mu \mathrm{g}$ protein) and were precipitated with trichloroacetic acid, washed with ethanol, and air dried. Samples were resolubilized in $70 \mu \mathrm{l}$ BUST at room temperature for several hours and at $90^{\circ} \mathrm{C}$ for $5 \mathrm{~min}$. Samples were electrophoresed and fluorographed as described above. Subunit "column" molecular weights for individual SCb polypeptides were assigned by comparison of elution volume with that of known standards.

\section{Results}

Distribution of $\mathrm{SCb}$ radioactivity with time. Figure 1 represents graphically the distribution of total radioactivity in the optic nerve, chiasm, and tract of the guinea pig visual system at 4,6 , and 9 days after intraocular injection of amino acids. As can be seen, the radioactivity drops off sharply with distance from the eye at 4 days, and there is a small leading foot at the base of the advancing $\mathrm{SCb}$ front. By 6 days, the front of radioactivity has advanced to the chiasm and the crest of the peak extends back several millimeters. By 9 days, the bulk of the radioactivity has moved to the tract regions and beyond. The average rate of movement of the initial peak segment of the radioactive profile was approximately 2 $\mathrm{mm} /$ day.

Characteristic SCb polypeptides. An SDS-polyacrylamide gel fluorograph displaying the characteristic $\mathrm{SCb}$ polypeptide profile is shown in Figure 2. This radioactive profile was obtained by allowing 6 days to pass after intraocular radiolabeled precursor injection (when the

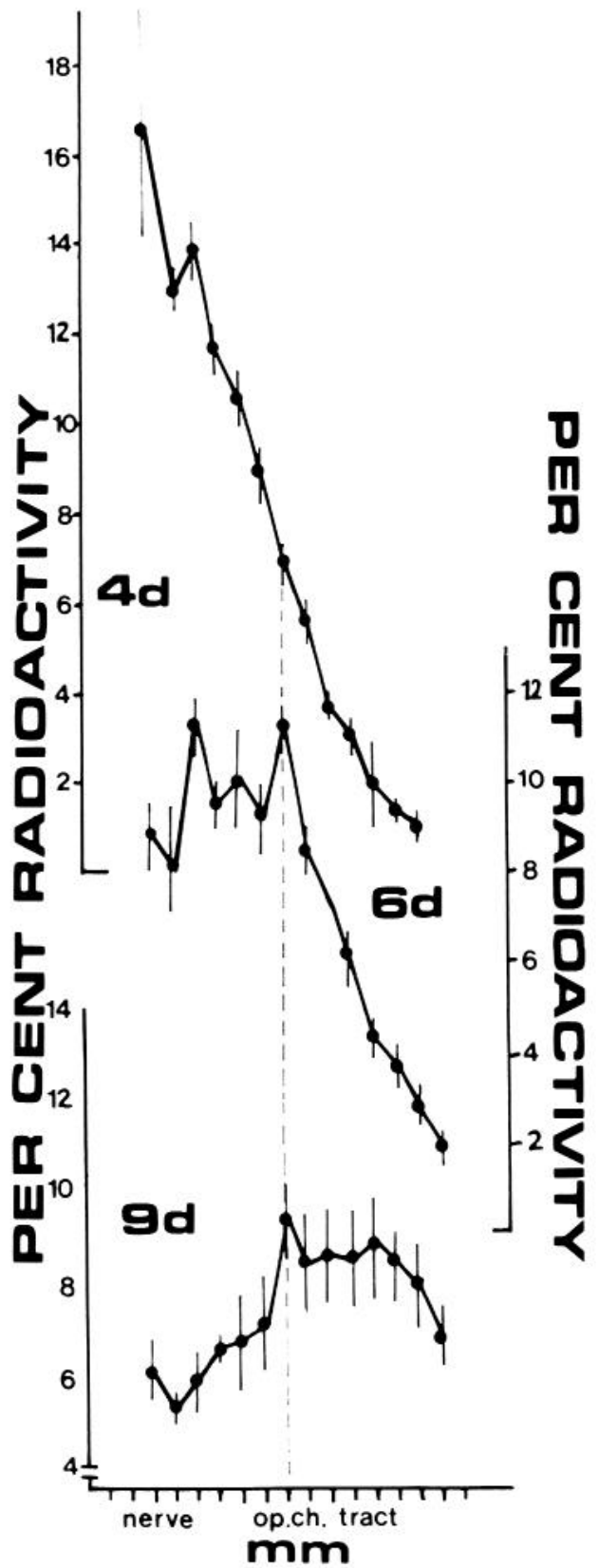

Figure 1. Distribution of radioactivity in the visual system at 4,6 , and 9 days after intraocular injection. At 4, 6, and 9 days after intraocular injection of ${ }^{3} \mathrm{H}$-radiolabeled amino acids, the optic nerves, chiasma, and tracts were harvested from experimental guinea pigs. The optic systems were cut into $1-\mathrm{mm}$ segments and solubilized, and the radioactivity was determined. The percentage of radioactivity was determined as described in the text and is presented as percentage of radioactivity per $\mathrm{mm}$ of optic system. The number of optic systems included is 8 at 4 days, 10 at 6 days, and 5 at 9 days.

initial SCb peak is present within the guinea pig optic chiasm). The molecular weights of the major polypeptides range from 17,000 to $>500,000$ with the majority between 25,000 and 100,000. Quantitative analysis of the radioactivity in the gels demonstrates that the great majority of radioactivity is present in the polypeptide bands at $500,000,56,000,48,000$ to $50,000,39,000$ to 42,000 , 35,000 , and 33,000 . 


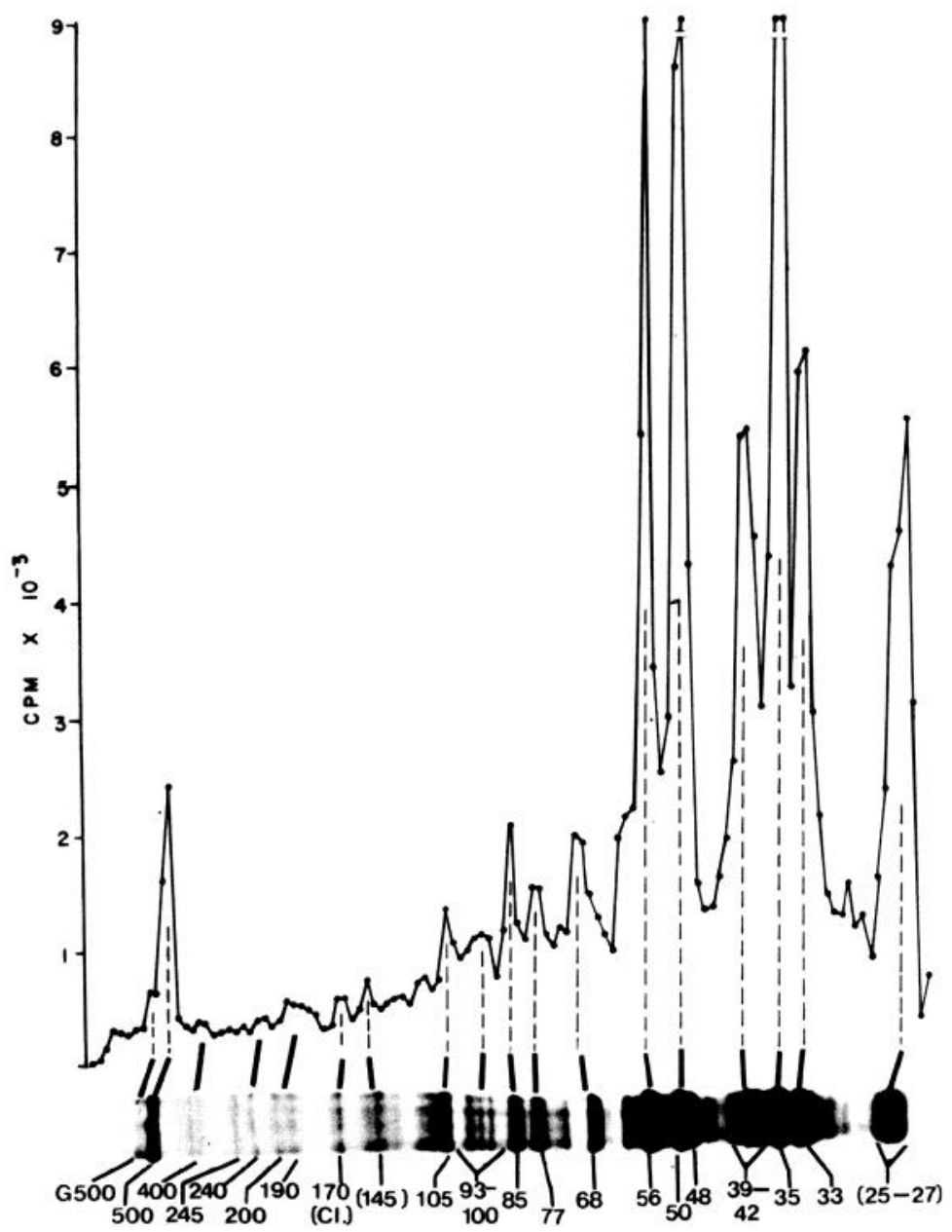

Figure 2 . SCb polypeptides: SDS-PAGE profile and relative radioactivity. At 6 days after intraocular injection of radiolabeled amino acids, an optic chiasm was prepared for electrophoresis as described in the text. Identical lanes were run on an SDS slab gradient (4 to $17.5 \%$ ) polyacrylamide gel. The fluorograph of one lane is shown horizontally with the major SC4 polypeptides indicated by their approximate molecular weight. The other lane was cut into $1-\mathrm{mm}$ pieces and dissolved, and the radioactivity in each $\mathrm{mm}$ was determined. The quantitative radioactive profile then was plotted against distance migrated within the SDS gel. G500 refers to the polypeptide with $M_{\mathrm{r}}>500,000$ and $\mathrm{Cl}$. indicates the putative clathrin band. Polypeptides with molecular weights in parentheses were not analyzed, as stated in the text.

Properties of the SCb polypeptides. In order to test preliminarily the cohesiveness of the $\mathrm{SCb}$ polypeptides in vitro, optic nerve and chiasm segments containing only radioactive $\mathrm{SCb}$ polypeptides were homogenized in a minimal amount of buffer, centrifuged, and then subjected to Sephadex G-200 liquid chromatography under nondenaturant conditions. It was found, initially, that only 25 to $30 \%$ of the $\mathrm{SCb}$ radioactivity was found in the supernatant of a $100,000 \times g$ spin. Preliminary experiments in which various detergents were used during the homogenization procedure indicate that the pelleting of the major portion of $\mathrm{SCb}$ radioactivity is not a result of simple trapping (Heriot, 1981). This observation is consistent with the idea that the major portion of $\mathrm{SCb}$ polypeptides is part of a structural assembly. The "soluble" $\mathrm{SCb}$ polypeptides in the supernatant fraction were analyzed by gel filtration, and the individual fractions were compared by SDS-PAGE. Even under mild nondenaturant conditions, most of the $\mathrm{SCb}$ polypeptides found in the supernatant fraction behave as monomers upon gel filtration; they elute at molecular weights corresponding to their subunit molecular weights in SDSpolyacrylamide gels in the presence of reducing agents. Some SCb polypeptides, however, do have column molecular sizes that are approximate multiples of their subunit molecular weights $(93,000$ to $100,000,95,000$, $56,000,39,000$ to 43,000 , and 35,000 ). It is possible that these polypeptides are dimeric or trimeric in their "soluble" form.

Anterograde movement of radiolabeled $S C b$ polypeptides: Fluorographic representation. When the guinea pig optic systems are cut into 1-mm segments at 4,6,9, 


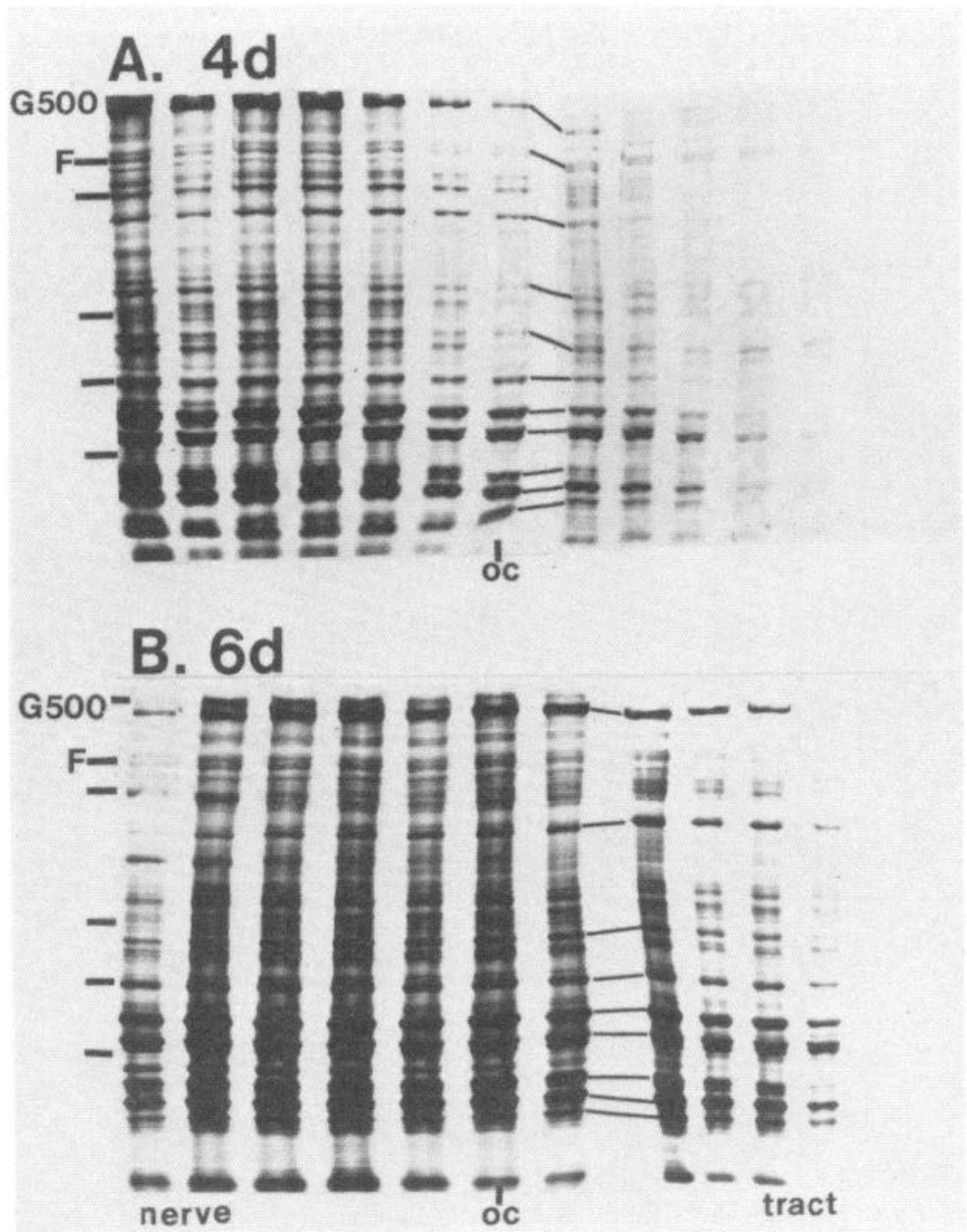

Figure 3. Fluorographic analysis of the movement of $\mathrm{SCb}$ polypeptides at 4 and 6 days after intraocular injection. At $4(A)$ and $6(B)$ days after intraocular injection of radiolabeled precursors, guinea pig optic systems were cut into sequential 1-mm segments and were electrophoresed in order on 4 to $17.5 \%$ gradient slab SDS-polyacrylamide gels and then fluorographed as described in the text. The gel lanes farthest to the left had sample profiles characteristic of segments closest to the guinea pig retina. The initial optic chiasm segment was used as a reference point and is indicated by $o c$. The bars at the left indicate the relative mobility of molecular weight markers at 200,000,170,000,94,000, 68,000, and 46,000. The presence of the fodrin doublet (Skene and Willard, 1981) is designated by $F$.

and 12 days after intraocular injection of protein precursors, and the individual segments are run sequentially on SDS-polyacrylamide gels, then fluorographed, as shown in Figures 3 and 4, one can observe the change in distribution of all the labeled $\mathrm{SCb}$ polypeptides with time. Although fluorographs made from the SDS gels are not quantitative, relative increases and decreases in radioactivity are reflected by changes in intensity of the bands.

If the general distribution of $\mathrm{SCb}$ radioactivity, rather than that of any one polypeptide, is examined at the four times indicated in Figures 3 and 4, a change in intensity of radioactivity can be seen in the shift from left (proximal to retina) to right (distal) with increase in time. At 4 days (Fig. $3 A$ ), the intensity of the radioactivity falls rapidly in the "front" region (through the optic chiasm into the optic tract). The individual polypeptide bands also decrease in radioactive intensity distally, mimicking the general "front" (with a few exceptions to be noted later). At 6 days (Fig. $3 B$ ), the most intensely labeled profiles extend into the visual system as far as the first optic chiasm segment, distal to which the intensity of the bands drops sharply.

At 9 and 12 days (Fig. 4, $C$ and $D$ ), the $\mathrm{SCb}$ radioactivity extends through the entire visual system, while the most intensely labeled profiles are in the optic tract. At 9 days, the $\mathrm{SCb}$ front has progressed into and beyond the optic tract. At 12 days, the peak has passed into the terminal regions and only the trailing edge can be seen. 

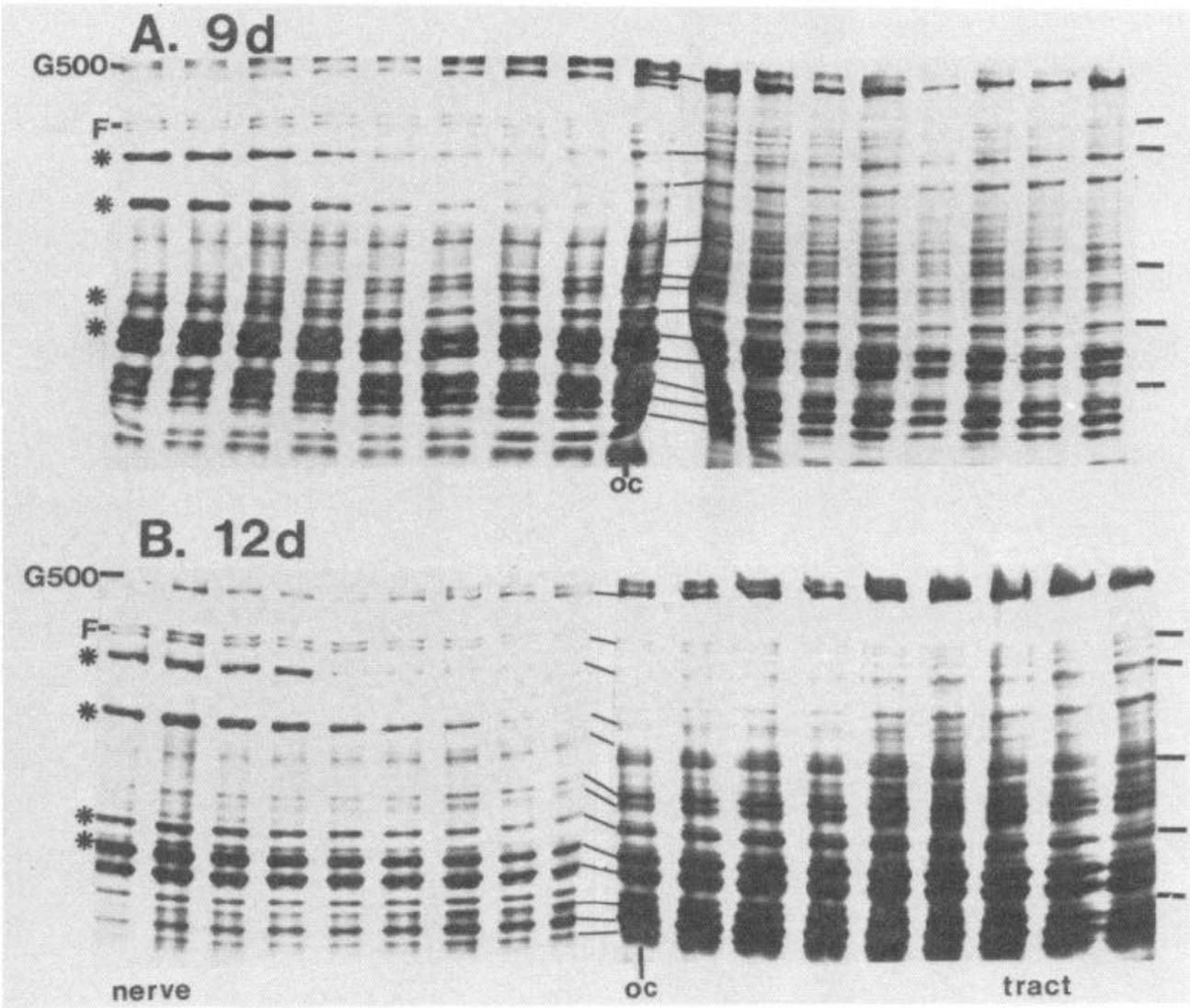

Figure 4. Fluorographic analysis of the movement of $\mathrm{SCb}$ polypeptides at 9 and 12 days after intraocular injection. At $9(A)$ and $12(B)$ days after intraocular injection of radiolabeled precursors, guinea pig optic systems were harvested and treated as described in the legend of Figure 3 and in the text. The bars at the right indicate the relative mobility of molecular weight markers at $200,000,170,000,94,000,68,000$, and 46,000 , and $F$ demonstrates the migration of fodrin. The asterisks at left indicate the relative mobility of the five major SCa polypeptides (they can be seen entering the optic nerve at 12 days postinjection particularly well).

Some of the polypeptide bands have distributions that appear to deviate slightly from the general pattern. At 4 and 6 days, a few of the bands (at 400,000, 245,000, $240,000,77,000,48,000$ to 50,000 , and 35,000 ) have distributions that extend slightly in advance of the remainder of the SCb bands. At 9 and 12 days, the more lightly labeled polypeptide bands and the 500,000 band appear to decline in intensity at a slightly sharper rate than do the more heavily labeled polypeptides. In addition, the $>500,000$ band is interesting because it increases in intensity slightly behind the rest of $\mathrm{SCb}$, suggesting that the $>500,000$ protein is out of phase with the $\mathrm{SCb}$ wave as a whole. Subjective analyses of fluorographs are insufficient for accurate descriptions of radiolabeled polypeptide distributions within the optic axons. Thus, direct quantitation of the radioactive distributions was undertaken.

Individual SCb polypeptide distributions at 4, 6, and 9 days: Quantitative analyses. Three major criteria were employed to select polypeptide bands for quantitative analysis. First, there had to be sufficient radioactivity within a polypeptide band to make the analysis feasible. The lowest acceptable limit for quantity of radioactivity within a band at it's peak in the axon was set at $\sim 300$ cpm. (Maximum cpm per polypeptide band ranged between $10^{4}$ and $10^{5} \mathrm{cpm}$.) Second, those polypeptide bands with relative mobilities in gels that were similar to those of the major $\mathrm{SCa}$ polypeptide bands were eliminated to avoid confusion. Third, only those SCb bands that were physically possible to excise reproducibly were chosen.

Two-dimensional gel electrophoresis of the $\mathrm{SCb}$ polypeptides, shown in Figure 5, reveals that several of the polypeptides, those at $>500,000,500,000,170,000$, and 85,000 , are most likely single polypeptide species, as only a single spot was generated in the second dimension. However, it also can be seen that many of the $\mathrm{SCb}$ polypeptides contain minor species (or multiple spots), indicating that the one-dimensional polypeptide band actually is comprised of several distinct polypeptides. An attempt was therefore made, in this study, to use as many polypeptides as possible having only a single major spot (that is, containing over $50 \%$ of the radioactivity) in two-dimensional gels. In the case of some $\mathrm{SCb}$ bands (i.e., 39,000 to 42,000 and 93,000 to 100,000 ), multiple bands were used knowingly, the rationale being that gross differences in distribution of the individual bands in a weight class at different times probably would be reflected in the distribution of that polypeptide "group." 


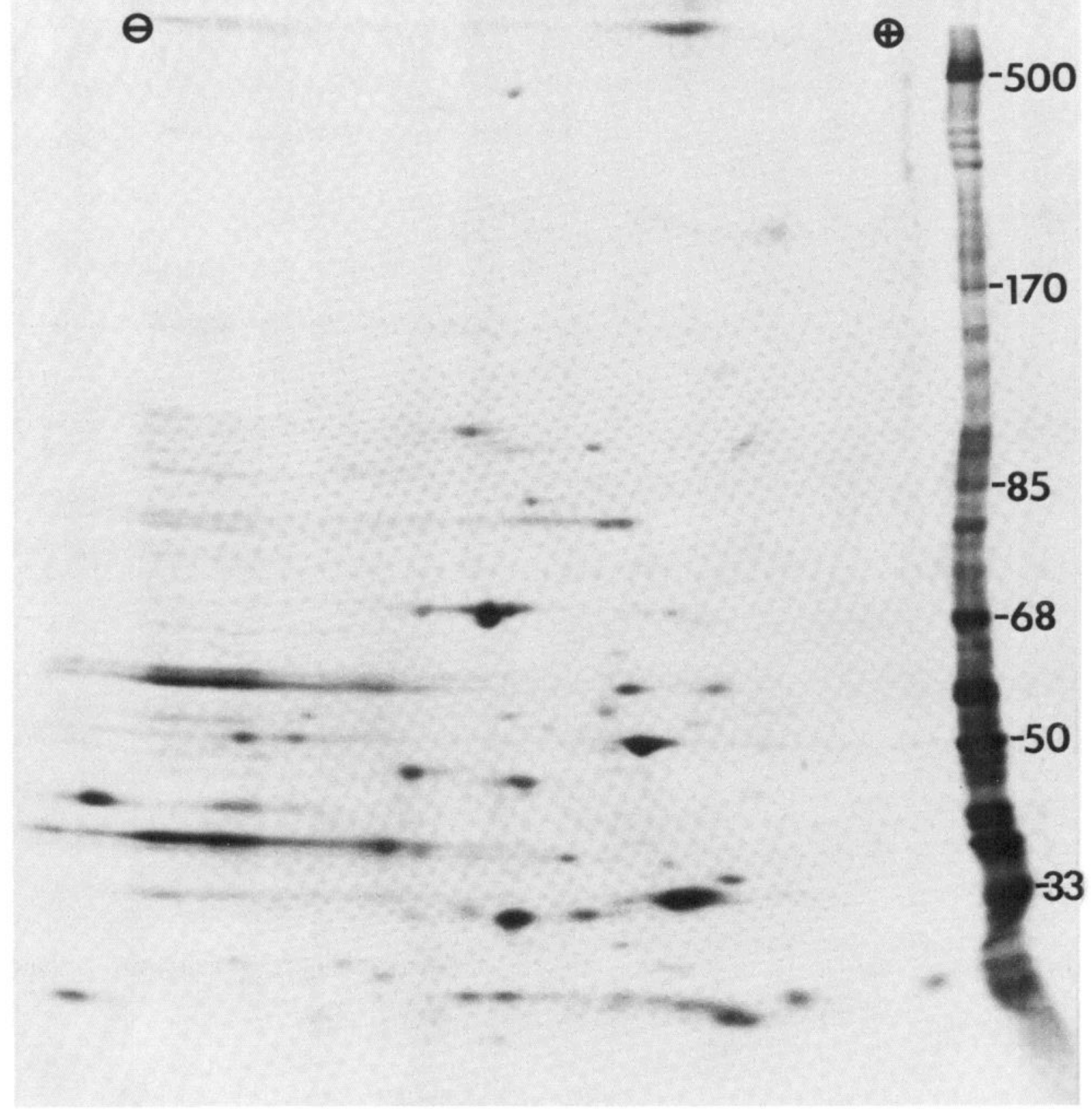

Figure 5. Two-dimensional gel electrophoresis of the SCb polypeptides. Samples containing radiolabeled SCb polypeptides were subjected to isoelectric focusing in the first dimension, then electrophoresed on 4 to $17.5 \%$ gradient slab polyacrylamide gels in the second dimension. The two-dimensional gels were fluorographed as described in the text. The single gel lane is an identical sample of radiolabeled $\mathrm{SCb}$ electrophoresed concurrently with the second dimension. Acidic and basic portions of the gel and major SCb polypeptide bands are as indicated.

The radiolabeled bands finally selected for quantitative analysis were $>500,000,500,000,400,000,245,000,240,000$, $200,000,190,000,170,000$ (putative clathrin band), 105,$000,93,000$ to $100,000,85,000,77,000,68,000,56,000,50$,$000,48,000,39,000$ to $42,000,35,000$, and 33,000 . Radiolabeled bands at relative mobilities of 400,000, 245,000, 240,000 , and 190,000 were only partially characterized as there was insufficient radioactivity associated with these bands to obtain reproducible results.
In order to determine the actual distribution of individual $\mathrm{SCb}$ radiolabeled bands with time in a quantitative manner, the radioactive bands were cut from the gels, dissolved, and counted directly as described under "Materials and Methods." It is impractical to present a catalogue of the distribution of all of the individual $\mathrm{SCb}$ bands because of space limitations (they are published elsewhere; Garner, 1979). To simplify the comparison, we have superimposed the individual distribution profiles at 
each of the three time points (Fig. 6). It can be seen, at 4 days after intraocular injection, that the "front" regions of the individual polypeptide distributions drop off very sharply distal to the initial optic nerve segments. At 6 days, the crest of the advancing "front" radioactivity has reached the initial optic chiasm segment, then declines distally at an angle mimicking the "front" regions seen at 4 days. At 9 days, the "front" regions have become somewhat more diffuse in distribution relative to each other; however, in general, the polypeptide bands are still more similar in the "front" profile than in the profiles trailing behind the peak. Linear regression analysis was performed on those segments directly below the horizontal line labeled LR in Figure 5. The slope of any one linear regression line was well within one standard deviation of the average slope for all of the polypeptides. In addition, if the mean 4-day "front" slope is compared to the mean 6-day "front" slope, they are within one standard deviation of each other. This suggests that the distributions of the individual polypeptide bands are extremely similar at the advancing "front" region as the $\mathrm{SCb}$ wave travels down the axon.

Examination of the portions of the radioactivity distributions trailing behind the peak of moving material at 6 and 9 days, however, reveals considerable variation in rapidity of decline to base line radioactivity. This variation ranges from a very sharp decline after the peak (see arrows at 6 days in Fig. 6) to the more generally seen broad crest and slow decline. Interestingly, the same polypeptide bands exhibiting the most widely variant trailing profile at 6 days are the same bands exhibiting the most widely variant profiles at 4 days, also indicated with arrows.

Comparison of the distribution of the individual radioactive polypeptides (with particular attention paid to the trailing edges of the distribution curves) led us to the conclusion that there were at least three different classes of distribution profile exhibited by the SCb polypeptides.
The three representative profiles will be designated classes I through III, with classes I and III being the most extreme in their difference. In Figure 7, individual polypeptide distributions that are most representative of the three classes are presented. In addition, Table I lists the other $\mathrm{SCb}$ polypeptides having distributions that can be categorized into one of the three classes. Class I distributions (possessed by the largest number of SCb polypeptides) are characterized by a broad peak region and a relatively slow decline in radioactivity behind the peak in the trailing portion of the curve (see the 6-day and 9day profiles of the $M_{\mathrm{r}}=85,000$ polypeptide in particular). Class III distributions (illustrated by the $M_{\mathrm{r}}=500,000$ profiles), on the other hand, have sharp peaks and decline rapidly in radioactivity at base line levels directly behind the peak, presenting an almost triangular distribution profile as the polypeptide travels down the axon. The slopes of the trailing portions of the class I and class III curves exhibit a statistically significant difference when subjected to the Student's $t$ test.

Class II distributions (as in those of the $M_{\mathrm{r}}=33,000$ polypeptide) appear different from the other two classes in that they exhibit a "sawtooth" profile that is particularly noticeable in the 6-day profiles. Class II profiles also have broad pcaks and decline fairly slowly to base line.

Although electrophoresis in two dimensions reveals the actual complexity of the individual polypeptide bands in the one-dimensional profiles (Fig. 5), we have confidence that these three distribution classes are individually distinct for the following reason. In each class there is at least one polypeptide in which the overwhelming majority of radioactivity focuses as a single spot after two-dimensional electrophoresis. For example, "single" polypeptides at $85,000,33,000$, and 500,000 are all characteristic of classes I, II, and III, respectively. In addition, all three classes contain both lightly and heavily labeled $\mathrm{SCb}$ bands. This suggests that the three different distribution classes probably would not be due to additive
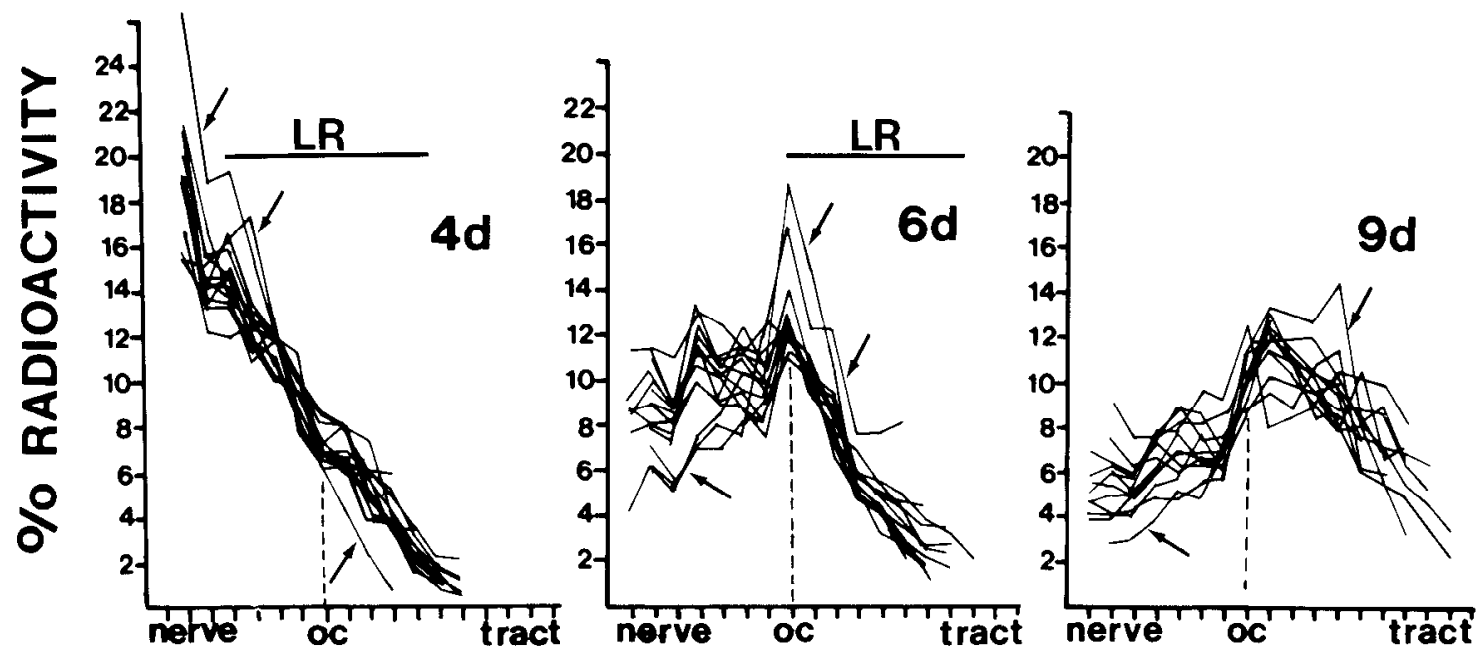

Figure 6. Distribution curves of all of the individual radiolabeled $\mathrm{SCb}$ polypeptides superimposed at each time point after intraocular injection. The distribution of the individual radiolabeled SCb polypeptides was determined and standardized as described under "Materials and Methods." The individual distributions at each time were superimposed to show relative similarity of distribution profiles. The segments closest to the retina are to the left, and the initial optic chiasm segment is labeled oc to facilitate orientation. Linear regression lines were computed for the individual polypeptides in the segments directly below the line labeled $L R$, and the slopes of the regression lines were compared (at 4 and 6 days). Distribution curves most widely varying in distribution profile are indicated with arrows (they belong to the same polypeptides at each time: 500,000 and 170,000). 

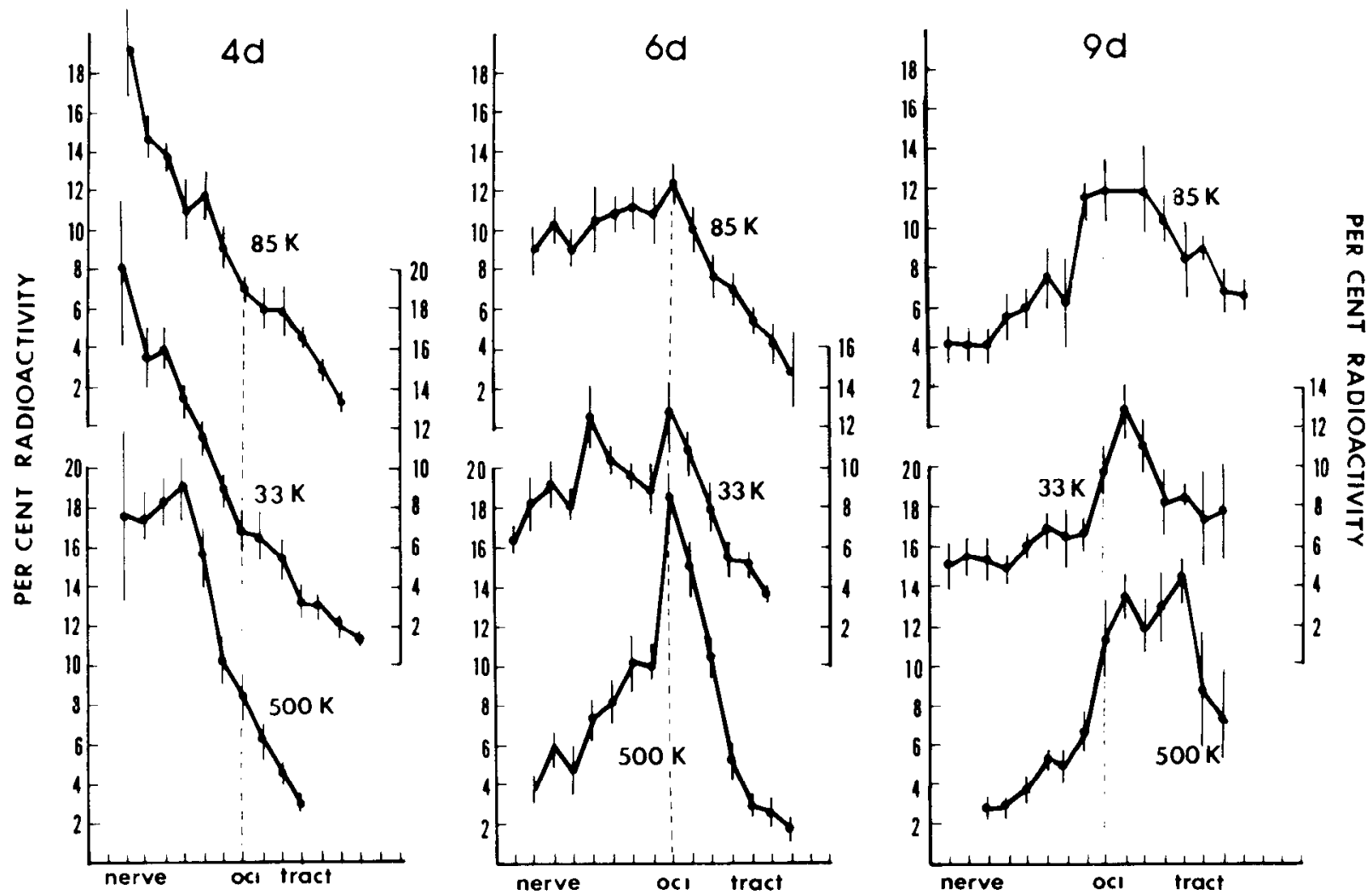

Figure 7. The three general "classes" of distribution curves for the SCb polypeptides. The distribution profiles of the three radiolabeled SCb polypeptides most representative of the three distinct "classes" of distribution profile are presented at 4, 6 , and 9 days after intraocular injection. The $M_{r}=85,000$ polypeptide is representative of the class I distribution, the $M_{\mathrm{r}}=33,000$ polypeptide represents the class II or "sawtooth" distribution and the $M_{\mathrm{r}}=500,000$ polypeptide represents the class III distribution. The initial optic chiasm segment is indicated by the dashed lines. The vertical bars through the points show the standard crror.

TABLE I

$S C b$ polypeptides categorized according to distribution class Polypeptides at 400,000,245,000,240,000, and 190,000 were not included due to low radioactivity.

\begin{tabular}{ccc}
\hline Class I & Class II & Class III \\
\hline$>500,000$ & 77,000 & 500,000 \\
$93,000-100,000$ & 68,000 & 200,000 \\
85,000 & $48,000-50,000$ & 170,000 \\
$39,000-42,000$ & 33,000 & \\
35,000 & 105,000 & \\
\hline
\end{tabular}

effects of multiple band distributions that differ significantly in shape. Several $\mathrm{SCb}$ polypeptides (at 400,000 , $245,000,240,000$, and 190,000) were not included in this analysis because of insufficient radioactivity; however, preliminary examination of their 6-day profiles indicates that each of them probably falls into one of the classes mentioned above. In addition, it is interesting that if one re-examines Figure 6 with the three classes in mind, the distribution profiles that are most different from the majority of the distribution curves (designated with $a r$ rows) are those belonging only to class III.

\section{Discussion}

SCb as a structural assembly. The evidence presented in this study strongly supports the idea that the SCb polypeptides form and are transported as a structural assembly in the axons, rather than simply moving toward the terminals independently. The evidence is provided by examination and comparison of the radioactive distributions of the individual $\mathrm{SCb}$ polypeptides at three times during their transport in the visual system. The distributions exhibit a great degree of coherence in the "front" regions advancing toward the axon terminals and in the location of the initial peak or crest portions of the individual distribution profiles over both time and distance. Since the advancing "front" must represent the average maximum rate at which an individual polypeptide moves, it must be concluded that polypeptides that have nearly superimposable front and crest regions are traveling at the same rate. In this dynamic system, a large number of individual polypeptides move a great distance in the same direction at the same rate. It seems most probable, in view of the similarity of their distribution profiles, that they achieve this phenomenon by being moved as a supramolecular structural assembly. The postulate that the $\mathrm{SCb}$ polypeptides form a structural assembly is further supported by previous centrifugation studies (Lorenz and Willard, 1978; Levine and Willard, 1980) and the ones described in this study. In all of these studies, it was found that the $\mathrm{SCb}$ polypeptides tend to act like a "complex" (i.e., most SCb radioactive polypeptides are found pelleted together at fairly high centrifugal force rather than in the cytosol, where independent "soluble" 
polypeptides would be expected to be). In addition, recent studies by Heriot (1981) have shown that all SCb polypeptides pelleted at $10,000 \times g$ migrate in a single density band after isopycnic centrifugation on discontinuous sucrose gradients.

The alternative explanation for the results we have observed would be that all of the $\mathrm{SCb}$ polypeptides share some chemical or hitherto unknown property that would allow them to be transported without interassociations. We feel that such an explanation, though possible, is unlikely because logically, bulk flow mechanisms (without interassociation) would be much more likely to allow spreading or other changes in the total distributions of the individual polypeptides, particularly over time. That is, if all of the polypeptides are moving independently, their distributions probably would not exhibit what is observed: statistically indistinguishable distributions in the advancing slopes at both 4 and 6 days. In addition, the unlikelihood of the bulk flow mechanism is supported by the lack of change in the slope of the "fronts" between 4 and 6 days. To make our argument even more convincing, of course, requires detailed analysis of the $\mathrm{SCb}$ polypeptides in a much longer system.

Complexity of the SCb polypeptide interactions within the structural assembly. While the initial leading portions of the individual SCb polypeptide waves are nearly identical in location and shape over both time and distance, there is a great degree of variation among the individual polypeptides in the rapidity with which the radioactivity trailing behind the peak returns to background levels. In light of our hypothesis, we can interpret these results in the following way. The $\mathrm{SCb}$ polypeptides have nearly identical distribution at the "front." If they are moving as a supramolecular structure, this would allow a large and diverse population of polypeptides to move as a unit through the axoplasm. Second, in this case, the variation in trailing regions could be accounted for by differences in each individual polypeptide's specific binding affinity for the $\mathrm{SCb}$ component as a whole. For instance, if a polypeptide were to have a relatively broad peak and its trailing profile declined to background gradually, this would indicate relatively low affinity for the moving SCb structure. Alternatively, a polypeptide that demonstrates the most sharp peak would appear to have a relatively high affinity for the $\mathrm{SCb}$ complex. Once an $\mathrm{SCb}$ polypeptide has disengaged from the SCb complex, it could only follow one of two courses. First, once released, it might not interact with anything in the axoplasm and its subsequent movement would be governed by the laws of diffusion alone. A second possibility would be that the $\mathrm{SCb}$ polypeptide could interact with constituents of another moving component. This could include interactions with members of the fast component, the intermediate components, the more slowly moving $\mathrm{SCa}$ component, or the material moving somatofugally in the retrograde component or a new interaction with the $\mathrm{SCb}$ complex. Examination of the individual $\mathrm{SCb}$ polypeptide distributions reveals that one of these possibilities may occur. If one superimposes an individual polypeptide distribution over that of the total $\mathrm{SCb}$ radioactivity distribution, the polypeptide may slightly precede the $\mathrm{SCb}$ wave (as in Garner and Lasek, 1981; Mori and Kurokawa,
1981; with the clathrin distribution), although this procedure was not statistically significant in our previous study. This may suggest transient interaction between an SCb polypeptide (such as clathrin) and a more rapidly moving component.

The $\mathrm{SCb}$ polypeptides have distributions that fall into three distinct classes and cover the entire range of possible molcular interactions predicted by our hypothesis. Class III distributions are sharply triangular in shape and do not appear to diminish in height or increase in breadth as they travel down the axon. This suggests that the class III polypeptides have very strong affinities for the putative $\mathrm{SCb}$ complex and therefore do not disengage in significant amounts. In fact, the stability of these poly. peptides suggests a possible role for them as a "backbone" macromolecule for the SCb structural complex. It is interesting to note that one of the identified polypeptides, clathrin, has a distribution profile that falls within this class.

The class I polypeptides are at the other end of the distribution spectrum; they have a very broad peak and the material trailing behind the peak descends very gradually. The peaks appear to broaden as they move, suggesting that the polypeptides with class I distributions (such as actin; Black and Lasek, 1979) would be able to "disengage" from the putative SCb complex more readily than their counterparts in class III. The slight differences among the class I polypeptide distributions in degree of material trailing behind the moving peak might reflect the strength of their affinity for the complex.

The remaining class, class II, or "sawtooth" profile, is a double peak with a gradual decline in radioactivity trailing behind the peak. It appears, in this case, that these polypeptides are traveling in two waves: the first one coincident with the class I SCb peak and the second one traveling slightly slower than the main body of the $\mathrm{SCb}$ wave. This finding suggests that there may be "subcomponents" of the SCb group of polypeptides that disengage from the main $\mathrm{SCb}$ group, then interact with a more slowly moving component/structure. The observation of different classes of distribution suggests that, if our hypothesis is correct, there may be an entire range of distribution profiles possibly due to the presence of a large number of structural SCb "subcomplexes" (Lorenz and Willard, 1978; Levine and Willard, 1980; Heriot, 1981).

An alternative explanation for the above interpretation could account for the observed variation in trailing distribution. If the $\mathrm{SCb}$ polypeptides did form a structural complex and the structural complex were a very static one, the observed differences in trailing portions of the radiolabeled polypeptide distribution could be a result of differential introduction of the pulse-labeled species into the structural assembly at the point of its synthesis. If the above explanation were the case, one would expect to see no change in trailing distribution as the complex moved down the axon. Although a definitive answer to this question can again only be provided by a similar study in a much longer system, we feel that this explanation is made less likely by the exhibited difference in the distribution in the trailing portions of the curves of each polypeptide between 6 days and 9 days. 
Criteria for inclusion of a polypeptide within a component of axonal transport. Because of the information concerning individual protein:protein interactions provided by a detailed analysis of the axonal transport of a group of polypeptides, it becomes increasingly important to define rigorously the criteria used for inclusion of a polypeptide within a particular rate component of axonal transport. Many axonal transport studies have utilized the "single window" technique to study the members of a particular axonal transport component. (This technique involves selecting a segment at uniform distance from the cell bodies and examining the polypeptides that move into and out of the segment by choosing appropriate times after presentation of radiolabeled amino acids to the cell bodies.) It can be seen that if one were to miss the peak of a particular polypeptide through inadvertently choosing an inappropriate time, particularly a polypeptide with a very sharply triangular (class III) distribution, that polypeptide would not appear to travel with the SCb component. At best, the behavior of a class III polypeptide would be highly anomalous in that it would appear to disappear prematurely or that polypeptides with long trailing distributions would "stay" within a segment for abnormally long periods of time. In fact, either of these possibilities may explain "discontinuities" previously noted in the transport of the $\mathrm{SCb}$ polypeptides (Levine and Willard, 1980).

It therefore appears that in defining a polypeptide's axonal transport, one must examine its entire distribution within a nerve, with maximum resolution (i.e., smallest possible segment) over time. It also underlies the need for a very careful analysis of the kinetics of a particular component. The minimum criterion for whether a particular polypeptide travels within a component would be that it moves "in an orderly way with other polypeptides within that component." The advancing "front" of a polypeptide would define when the polypeptide first can be resolved, and the "crest" would define when the bulk of that protein species passes. If a group of polypeptides first can be resolved at the same time, and the congruence of their peak regions is maintained as the polypeptide moves down the axon, this implies "movement in an orderly way with other polypeptides."

Conclusion. In conclusion, it is to be hoped that by defining the constituents of a rate component in the way we and others (Brady et al., 1981) have discussed, the normal interaction of the polypeptides will be indicated. It must be stated, however, that while all of our kinetic data are strongly supportive of our SCb structural assembly hypothesis, and many of the details can be easily interpreted in light of the hypothesis, it does not constitute proof that the $\mathrm{SCb}$ polypeptides are associated physically. In order to establish definitively that $\mathrm{SCb}$ is a supramolecular complex, biochemical studies aimed at physical isolation of the $\mathrm{SCb}$ complex itself must be performed. Preliminary centrifugation experiments such as those described above (Heriot, 1981) are a first step toward this goal.

\section{References}

Black, M. M., and R. J. Lasek (1979) Axonal transport of actin: Slow component $b$ is the principal source of actin for the axon. Brain Res. 171: 401-413.
Black, M. M., and R. J. Lasek (1980) Slow components of axonal transport: Two cytoskeletal networks. J. Cell Biol. 87: 616-623.

Bonner, W. M., and R. A. Laskey (1974) A film detection method for tritium labeled proteins and nucleic acids in polyacrylamide gels. Eur. J. Biochem. 46: 83-88.

Brady, S. T., and R. J. Lasek (1981) Nerve-specific emolase and creatine phosphokinase in axonal transport: Soluble proteins and the axoplasmic matrix. Cell 23: 515-523.

Brady, S. T., M. Tytell, K. Heriot, and R. J. Lasek (1981) Axonal transport of calmodulin: A physiologic approach to identification of long-term associations between proteins. J. Cell Biol. 89: 607-614.

Brimijoin, S. (1975) Stop flow: A new technique for measuring axonal transport and its application to the transport of dopamine- $\beta$-hydroxylase. J. Neurobiol. 6: 379-394.

Cancalon, P., and L. M. Beidler (1975) Distribution along the axon and into various subcellular fractions of molecules labeled with $\left[{ }^{3} \mathrm{H}\right]$ leucine and rapidly transported in the garfish olfactory nerve. Brain Res. 89: 225-244.

Droz, B., A. Ramboug, and H. L. Koenig (1975) The smooth endoplasmic reticulum: Structure and role in the renewal of axonal membrane and synaptic vesicles by fast axonal transport. Brain Res. 93: 1-13.

Forman, D. S., B. Grafstein, and B. S. McEwen (1972) Rapid axonal transport of ${ }^{3} \mathrm{H}$-fucosyl glycoproteins in the goldfish optic system. Brain Res. 48: 327-342.

Garner, J. (1979) Cohesive movement of polypeptides characteristic of a distinct component of axonal transport. Doctoral dissertation, Case Western Reserve University, Cleveland, $\mathrm{OH}$.

Garner, J. A., and R. J. Lasek (1978) Axonal transport kinetics of a distinct group of neuronal polypeptides. J. Cell Biol. 79: 103a.

Garner, J. A., and R. J. Lasek (1981) Clathrin is axonally transported as part of slow component $b$ : The microfilament complex. J. Cell Biol. 88: 172-178.

Grafstein, B., and D. S. Forman (1980) Intracellular transport in neurons. Annu. Rev. Physiol. 60: 1167-1253.

Gross, G. W., and L. M. Beidler (1975) A quantitative analysis of isotope concentration profiles and rapid transport velocities in the C-fibers of the garfish olfactory nerve. J. Neurobiol. 6: $213-232$

Heriot, K. (1981) Definition of the granulo-filamentous complex of the axon by biochemical and autoradiographic studies of the slow component $b$ of axonal transport. Doctoral dissertation, Case Western Reserve University, Cleveland, $\mathrm{OH}$

Hoffman, P. N., and R. J. Lasek (1975) The slow component of axonal transport. Identification of major structural polypeptides of the axon and their generality among mammalian neurons. J. Cell Biol. 66: 351-366.

Laemmli, U. K. (1970) Cleavage of structural protein during the assembly of the head of bacteriophage T4. Nature 277: 680 685.

Lasek, R. J. (1980) Axonal transport: A dynamic view of neuronal structure. Trends Neurosci. 3: 87-91.

Lasek, R. J., and P. N. Hoffman (1975) The neuronal cytoskeleton, axonal transport and axonal growth. In Cell Motility, R. Goldman et al., eds., Vol. 3, pp. 1021-1050, Cold Spring Harbor Laboratory, Cold Spring Harbor, NY.

Laskey, R. A., and A. D. Mills (1975) Quantitative film detection of ${ }^{3} \mathrm{H}$ and ${ }^{14} \mathrm{C}$ in polyacrylamide gels by fluorography. Eur. J. Biochem. 56: 335-341.

Levine, J., and M. Willard (1980) The composition and organization of axonally transported proteins in the retinal ganglion cells of the guinea pig. Brain Res. 194: 137-154.

Lorenz, T., and M. Willard (1978) Subcellular fractionation of intra-axonally transported polypeptides in the rabbit visual system. Proc. Natl. Acad. Sci. U. S. A. 75: 505-509. 
Mori, H., and M. Kurokawa (1981) Intra-axonal transport of clathrin and actin. Biomed. Res. 2: 677-685.

Ochs, S. (1972) Fast transport of materials in mammalian nerve fibers. Science 176: 252-260.

O'Farrell, P. H. (1975) High resolution two dimensional electrophoresis of proteins. J. Biol. Chem. 250: 4007-4021.

Schwartz, J. H., J. E. Goldman, R. T. Ambron, and D. J. Goldberg (1976) Axonal transport of vesicles carrying $\left[{ }^{3} \mathrm{H}\right]$ serotonin in the metacerebral neuron of Aplysia californica. Cold Spring Harbor Symp. Quant. Biol. 40: 83-92.

Skene, J. H. P., and M. Willard (1981) Characteristics of growthassociated polypeptides in regenerating toad retinal ganglion cell axons. J. Neurosci. 1: 419-426.

Tsukita, S., and H. Ishikawa (1980) The movement of membranous organelles in axons: Electron microscopic identification of anterogradely and retrogradely transported organelles. J. Cell Biol. 84: 513-530.

Tytell, M., M. M. Black, J. A. Garner, and R. J. Lasek (1981) Axonal transport: Each major rate component reflects the movement of distinct macromolecular complexes. Science 214: 179-181.

Weiss, P. A., and H. B. Hiscoe (1948) Experiments on the mechanism of nerve growth. J. Exp. Zool. 107: 315-395.

Willard, M. (1977) The identification of two intra-axonally transported polypeptides resembling myosin in some respects in the rabbit visual system. J. Cell Biol. 75: 1-11.

Willard, M., W. M. Cowan, and P. R. Vagelos (1974) The polypeptide composition of intra-axonally transported proteins: Evidence for four transport velocities. Proc. Natl. Acad. Sci. U. S. A. 71: 2183-2187.

Willard, M., M. Wiseman. J. Levine, and P. Skene (1979) Axonal transport of actin in rabbit retinal ganglion cells. J. Cell Biol. 81: 581-591.

Willard, M. B., and K. L. Hulebak (1977) The intra-axonal transport of polypeptide H: Evidence for a fifth (very slow) group of transported proteins in the retinal ganglion cells of the rabbit. Brain Res. 136: 289-306. 